\title{
Dynamic methods of identification of electromagnetic parameters of power transformers in non-stationary mode
}

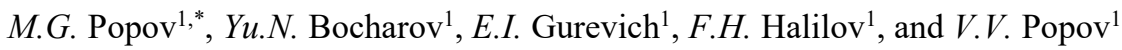 \\ ${ }^{1}$ Peter the Great St.Petersburg Polytechnic University, Polytechnicheskaya, 29, St. Petersburg, \\ 195251, Russia
}

\begin{abstract}
The report reflects the promising solutions for the creation of adaptive protection means and emergency automatics in the implementation of methods of identification of dynamic systems. Presented original research assigned at directly addressing the causes of the sensitivity lack of the transformer equipment protection - compensation for the magnetizing current of the power transformers by linearizing their transfer characteristics.
\end{abstract}

\section{The problem of identification of mathematical models parameters of power equipment}

Most of the numerical and analytical methods, used in practice for studying transients of electrical power equipment, are based on the use of its idealized (conservative) mathematical description and, as a result of this, inadequately reflect the real dynamic characteristics of the interconnected power systems. Initially, this caused by the methodological error due to the insufficient mathematical rigor of the formulation of the research problem, and in some cases in using of unreliable primary (initial) parameters of the mathematical model during the performance of numerical experiments. All this inevitably causes errors in solving the problem when performing computational studies and can lead to subsequent misinterpretation of the obtained results, which is ultimately reflected in the erroneous view of the operation reliability and stability of the power system [1-17].

As a rule, the idealization of mathematical models of electrical power equipment of the network is based on the use of concentrated complex equivalent circuits, whose linear parameters are calculated using known analytical expressions under the assumption of stationarity (single part) of the electromagnetic process. Moreover, in the conditions of a full-scale physical experiment with a new electrical power equipment, the idealization of adjacent elements of the power network is used. Quite often, power sources of the examined electrical equipment are idealized, thereby introducing methodological roughness and possible distortions of the experimental results. However, due to the unjustified

\footnotetext{
${ }^{*}$ Corresponding author: PopovMG@eef.spbstu.ru
} 
complication of the model and the inability to meet the computational performance requirements of modern microcontrollers, the use of a rigorous mathematical description of electrical power equipment transients may not be acceptable when solving the problem of improving and expanding of the functionality of microprocessor protection means and automatics [11-18].

In connection with these technical difficulties, the task of creating adaptive parametric mathematical models with a relatively simple structure that adequately reflects the dynamic properties of electrical power equipment in non-stationary operating modes of the electrical network becomes crucial. Refinement of electrical parameters for the adopted (specified) structure of the mathematical model of electrical power equipment is carried out using the methods of identification of dynamic systems.

\section{Identification of parameters of electrical power equipment of the electric power system in various applications}

Consider the task of identifying the electrical parameters $(R, L \& C)$ of some electrical power equipment (load, transformers, etc.) of an electrical network connected to the busbars of the electric power system (EPS). To do this, we use the classical mathematical description of transients of electric installations using instantaneous values of external (measured) and internal (model) electrical signals. At the same time, to simplify the form of writing identification equations, symbolic operators of differentiation $(p)$ and integration $(1 / p)$ of functions (electrical signals) in the time domain are used. Also for clarity, the adopted block diagrams of identification objects are given, and the determination of the $R L C$-parameters of a given model of electrical equipment is carried out as a result of solving a system of nonlinear differential and integral equations of its transient processes. Naturally, the mathematical description of the transients of the model corresponds to the actual connection scheme of the investigated electrical (electromagnetic) equivalent.

Identification of the parameters of electrical power equipment in such a formulation of the problem allows to perform the synthesis of adaptive, self-adjusting systems for monitoring and controlling the operating modes of the power system. At the same time, the control of electric power system can be performed as a function of change (deviation) of the identified $R L C$-parameters of the electrical network equivalent.

The structural scheme of the identification of electrical parameters of electrical power equipment is shown in Figure 1.

The scheme includes the main microprocessor modules of parametric identification. Naturally, its connection is made to its primary converters of electrical signals to measuring voltage transformers $T V G 1$ and $T V G 2$, as well as to measuring current transformers $T A .1$ and $T A .2$ and the corresponding secondary converters of electrical current signals (current sensors $i / u$, Figure 1) and voltages (voltage sensors $u / u$, Figure 1). Subsequently, the electrical signals of voltage and current are subjected to analog-to-digital conversion in the ADC modules (in Figure 1, ADC blocks). Digital voltage signals $(u)$ and current $(i)$, measured using an $\mathrm{ADC}$, are fed to a processor (CPU, Figure 1), where integral and differential conversions of digital current and voltage signals are performed $\left(1 / p^{(n)}\right.$ and $\left.p^{(n)}\right)$ blocks in Figure 1) in accordance with the mathematical formulation of the problem of parametric identification. The solution of the system of identification equations $E(X)$ with respect to the desired $R L C$ - parameters is carried out using the numerical gradient method in the controller ( $C P U$ module, in Figure 1), the essence of which is described below. 


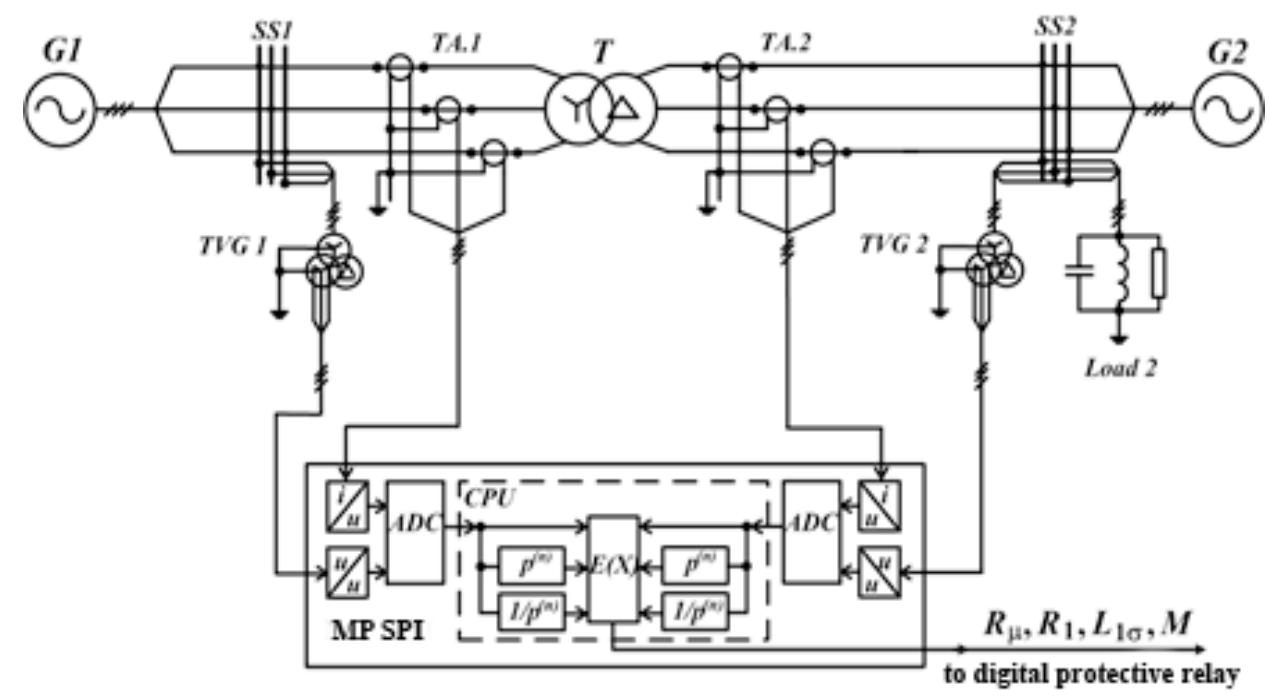

Fig. 1. Structural scheme of the identification subsystem of the parameters of adaptive current differential protection of the power transformer

Initially, we obtain the equations for the identification of electrical parameters of the Tshaped structure of the model of a two-winding power transformer of the TDC-125 000/110 brand $\quad\left(R_{1}=0.1124 \Omega ; \quad R_{2}=0.2624 \Omega ; \quad X_{\mu}=21.284 \mathrm{k} \Omega ; \quad X_{L 1}=1.23 \Omega\right.$; $\left.X_{L 2}=12.293 \Omega,[1,2]\right)$ :

$$
F(X)=\left\{\begin{array}{l}
u_{1}=i_{1} R_{1}-\frac{X_{L 1}}{\omega} p i_{1}+\left(i_{1}-i_{2}\right) R_{\mu}+\frac{X_{\mu}}{\omega}\left(p i_{1}-p i_{2}\right) ; \\
u_{2}=i_{2} R_{2}-\frac{X_{L 2}}{\omega} p i_{2}+\left(i_{2}-i_{1}\right) R_{\mu}+\frac{X_{\mu}}{\omega}\left(p i_{2}-p i_{1}\right) ;
\end{array}\right.
$$

where $R_{1}, R_{2}$ - the active resistances of the primary and secondary windings of the transformer, reduced to the nominal resistance and taken equal to 0.001 p.u. and 0.0022 p.u. respectively;

$X_{L 1}, X_{L 2}$ - inductive resistances of the scattering of the primary and secondary windings, reduced to the nominal resistance and taken equal to 0.011 p.u. and 0.110 p.u. respectively;

$R_{\mu}, X_{\mu}$ - the active and inductive resistances of the magnetizing branch of the transformer, reduced to the nominal resistance and taken equal to 500.0 p.u. and 181.713 p.u. respectively.

To reduce the obtained expressions (1) to a single dimension, we take as a basis the root-mean-square (effective) values: voltages $U_{b a s}$ and current $I_{b a s}$, from which base total power $S_{b a s}=U_{b a s} \cdot I_{b a s}$ and resistance $Z_{b a s}=\frac{U_{b a s}}{I_{b a s}}$ can be obtained. In addition, it is necessary to introduce the concept of the base frequency $\omega_{\text {bas }}=2 \pi f_{\text {bas }}$ and the integration step $d t=h$, that is inversely proportional to the sampling frequency of the ADC. However, it should be understood, that all variables in the identification equations, except for the base frequency $\omega_{b a s}$ and integration step $h$, are measured in relative units. Calculation (identification) of electrical parameters $X=\left(R, X_{L}, X_{C}\right)$ of the considered power 
transformers is made as a result of the numerical solution of the nonlinear system of equations in the matrix record form:

$$
\left[\frac{\partial E(t, X)}{\partial X}\right] \times[\Delta X]=-[E(t, X)]
$$

where $\frac{\partial E(t, X)}{\partial X}$ - the matrix of sensitivity coefficients, p.u.;

$\Delta X$ - the column vector of increments of the desired parameters, p.u.;

$E(t, X)$ - the column vector of the integral values of the objective function, defined by the expression, p.u.:

$$
E(X)=\sqrt{\frac{1}{T} \int_{t-T}^{T} F^{2}(X) \mathrm{d} \tau}
$$

where $T$ - the averaging interval, which is assumed to be $20 \mathrm{~ms}$.

In comparison with the objective function $F(X)$, its integral values $E(X)$ are signdefinite. Performing the transition according to (3), the final equations of the functions of the root-mean-square error $E_{i}(X)$ and its sensitivity are obtained:

$$
\begin{gathered}
E_{i}(X)=\sqrt{E_{i}^{2}(X)} ; \\
\frac{\partial E_{i}(X)}{\partial X}=\frac{0.5}{E_{i}(X)} \cdot \frac{\partial E_{i}^{2}(X)}{\partial X}
\end{gathered}
$$

The vector function $T \cdot E_{1}^{2}(X)$ for the transformer model after intermediate transformations will be determined by equation (4).

$$
\begin{aligned}
T \cdot E_{1}^{2}(X) & =\int_{t-T}^{t} u_{1}^{2} d \tau+R_{1}^{2} \cdot \int_{t-T}^{t} i_{1}^{2} d \tau-2 R_{1} \int_{t-T}^{t} u_{1} i_{1} d \tau+2 R_{1} \cdot \frac{X_{L 1}}{\omega} \cdot \int_{t-T}^{t}\left(i_{1} p i_{1}\right) d \tau+ \\
& +2 R_{1} \cdot R_{\mu} \cdot \int_{t-T}^{t} i_{1}\left(i_{1}-i_{2}\right) d \tau+2 R_{1} \cdot \frac{X_{\mu}}{\omega} \cdot \int_{t-T}^{t} i_{1}\left(p i_{1}-p i_{2}\right) d \tau+ \\
& +\frac{X_{L 1}^{2}}{\omega^{2}} \cdot \int_{t-T}^{t}\left(p i_{1}\right)^{2} d \tau-2 \frac{X_{L 1}}{\omega} \cdot \int_{t-T}^{t} p i_{1} u_{1} d \tau+2 R_{\mu} \cdot \frac{X_{L 1}}{\omega} \cdot \int_{t-T}^{t} p i_{1}\left(i_{1}-i_{2}\right) d \tau+ \\
& +2 \frac{X_{\mu} \cdot X_{L 1}}{\omega^{2}} \cdot \int_{t-T}^{t} p i_{1}\left(p i_{1}-p i_{2}\right) d \tau+R_{\mu}^{2} \cdot \int_{t-T}^{t}\left(i_{1}-i_{2}\right)^{2} d \tau+ \\
& +2 R_{\mu} \cdot \int_{t-T}^{t} u_{1}\left(i_{1}-i_{2}\right) d \tau+2 R_{\mu} \cdot \frac{X_{\mu}}{\omega} \cdot \int_{t-T}^{t}\left(i_{1}-i_{2}\right)\left(p i_{1}-p i_{2}\right) d \tau+ \\
& +2 \frac{X_{\mu}}{\omega} \cdot \int_{t-T}^{t} u_{1}\left(p i_{1}-p i_{2}\right) d \tau+\frac{X_{\mu}^{2}}{\omega^{2}} \cdot \int_{t-T}^{t}\left(p i_{1}-p i_{2}\right)^{2} d \tau .
\end{aligned}
$$


In order to reduce the basic materials, only one objective function and one of the thirtysix sensitivity equations are given, that are corresponding to the first diagonal element of the matrix $\frac{\partial E(t, X)}{\partial X}$ of $6 \times 6$ dimension.

$$
\begin{aligned}
\frac{\partial E_{1}}{\partial R_{1}}= & \frac{2 R_{1} \int_{t-T}^{t} i_{1}^{2} d \tau-\int_{t-T}^{t} u_{1} i_{1} d \tau+\frac{X_{L 1}}{\omega} \int_{t-T}^{t}\left(i_{1} p i_{1}\right) d \tau+R_{\mu} \int_{t-T}^{t} i_{1}\left(i_{1}-i_{2}\right) d \tau}{E_{1}}+ \\
& +\frac{\frac{X_{\mu}}{\omega} \int_{t-T}^{t} i_{1}\left(p i_{1}-p i_{2}\right) d \tau}{E_{1}}
\end{aligned}
$$

The presented above expression (5) is a power balance equation on the switching busbars of the primary winding of a two winding power transformer.

In accordance with the Kontorovich theorem [3], the convergence of the numerical solution of the nonlinear system of equations (2) largely depends on the initial approximation $X_{0}$. In addition, the speed of the solution depends on the presence of local extremes and surface ravine $E(X)$. Let us determine the qualitative surface character of the mean-square error $E(X)$ and its sensitivity to the initial values of the identification parameters $R$ ,$X_{L}$ and $X_{C}$ in the vicinity of the solution. Figure 2 shows the most characteristic dependences of the root-mean-square error in case of deviations of the initial values of the identification parameters of a two-winding transformer.

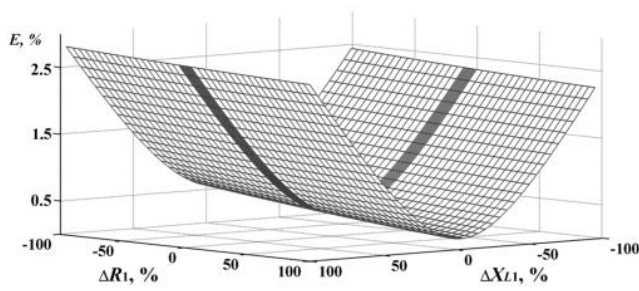

a) in the identification parameter space of primary winding R1 and XL1

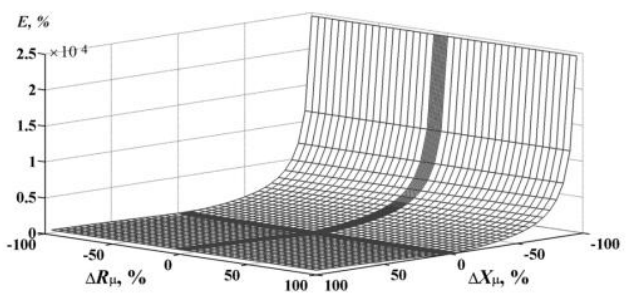

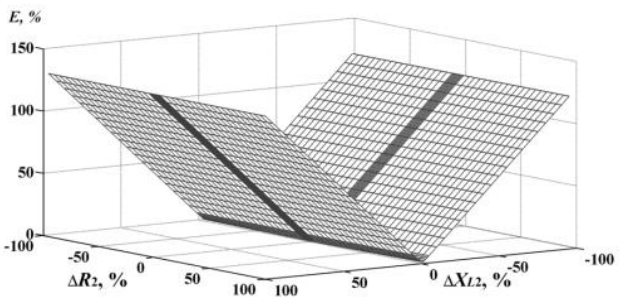

b) in the identification parameter space of secondary winding R2 and XL2

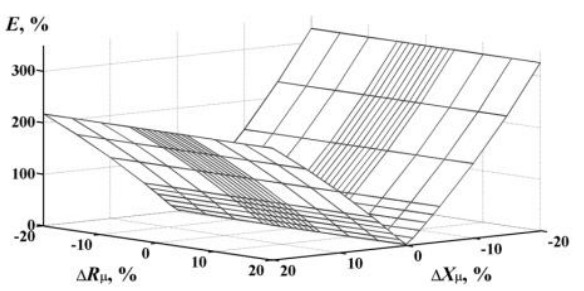

c) in the parameter space of the identification of the magnetization branch $R_{\mu}$ and $X_{\mu}$ (on the right the figure is in an enlarged scale)

Fig. 2. The nature of the change in the mean-square error $E(X)$ at variation of the initial values of the electrical parameters of the primary (a), secondary (b) windings and the magnetization branch (c) 
of a two-winding transformer.

Analysis of the results presented in Figure 2 showed a weak sensitivity of the identification equations to incorrectly specified initial conditions of the electrical parameters of the transformer primary winding. In particular, even with a 100\% error in setting the starting values, the root-mean-square error is no more than $3 \%$ (Figure 2, a), which guarantees the speed of the algorithm, even if the initial conditions are zero.

At the same time, the algorithm hypersensitivity to the specification of zero initial values of the electrical parameters of identification of the transformer magnetization branch was revealed. Under these starting conditions, the error reaches a value of more than 250 p.u. (Figure 2, c), which can lead to incorrect operation of the identification algorithm. To eliminate this defect, it is recommended to take overestimated initial values of the identified resistances of the magnetizing branch. In this region of positive deviations of the identification parameters, the surface of the vector function of the root-mean-square error is almost linear; its field lines monotonously decrease with respect to maximum values of about $250 \%$. The nature of the dependence of the vector function of the mean square error from the error of inaccurately specifying the initial resistances of the secondary winding is almost linear - the value of the error is proportional to the error of the initial conditions in modulus over the entire range of values under study.

To accelerate the convergence of the problem of identification of electrical parameters, it is recommended to use the second-order sensitivity equations (equations of "fastest descent"), which are used in gradient methods for solving systems of nonlinear equations.

\subsection{Testing of identification methods for power transformers under the physical experiments of the switching processes}

Testing of the developed algorithm for the identification of electrical parameters of transformer equipment was carried out when performing physical experiments on the switching of three-phase transformers in idle mode. When performing full-scale experiments, solution of several tasks was simultaneously performing - testing of methods and assessment of the effectiveness of algorithms for identification of electrical parameters of transformer equipment in non-stationary modes of their operation. Full-scale tests for the identification of electrical parameters were performed for the following transformers:

1. block three-phase two-winding transformers of type TC-630 000/330, with the scheme and connection group $\mathrm{Y} / \Delta-11$, installed at $330 \mathrm{kV}$ outdoor switchgear of the Leningradskaya NPP;

2. three-phase two-winding transformers of type TDC-80 000/110, TDC-125 000/110 and TD-40 000/110, with a scheme and connection group of windings $\mathrm{Y} / \Delta-11$ installed at $110 \mathrm{kV}$ substations of the $110 \mathrm{kV}$ distribution network of "JSC Lenenergo";

3. three-phase two-winding transformer with dry insulation of type Trihal $2500 / 10$, with a scheme and a connection group of windings $\Delta / Y-0-11$, installed in the Substation-14 of the distribution network of JSC Nissan Manufacturing Rus (St. Petersburg).

In connection with a significant amount of physical experiments materials, the most typical results of transients are described below with reference to the two-winding transformers of the TDC brand installed at $110 \mathrm{kV}$ substations of the $110 \mathrm{kV}$ distribution network of JSC "Lenenergo". The error assessment of the electrical parameters identification with the use of above described method was performed fully with respect to all the specified transformers and autotransformers.

Figure 3 shows digital oscillograms of phase currents of a power two-winding transformer TDC-80 000/110 $\left(\Delta P_{\text {UnLoad }}=85 \mathrm{~kW} ; I_{\mathrm{XX}}=0.55 \% ; \Delta P_{\text {ShortCircuit }}=320 \mathrm{~kW}\right.$; $U_{\text {ShortCicuit }}=11 \%$ ) in the switching mode on idling. However, the highest current value is 
typical for phase A, its instantaneous value is about 6.25 p.u. (Figure 3 ). In the subsequent stage of the non-stationary mode for about $0.5 \mathrm{~s}$ (Figure 3 ), the phase currents are damped to amplitude values close to the rated current (381.72 A). In the process of switching on, the start of the longitudinal current differential protection of the transformer was detected, the tripping of which was blocked by the acting of current filters of double frequency $(100 \mathrm{~Hz})$.

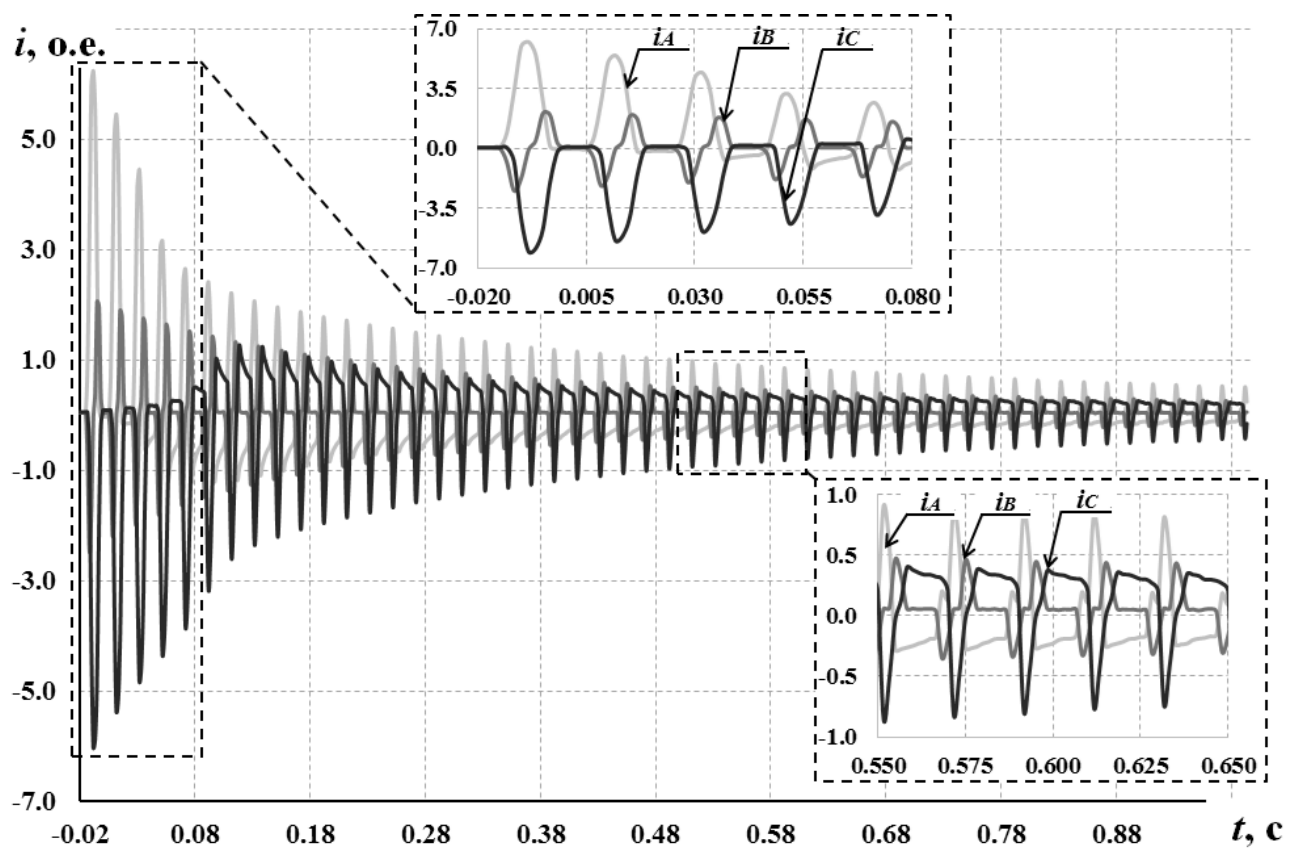

Fig. 3. Digital oscillograms of phase currents of switching the transformer TDC-80 000/110 in the idle mode

Digital oscillograms of full-scale experiments on the switching of the rest of the abovementioned list of power transformers are characterized by similar physical phenomena and do not have qualitative and fundamental features. In this case, the largest value of the magnetizing inrush current (about 6.25 p.u.) corresponds to the above-described results of a physical experiment. In other cases, the maximum values of the phase switching currents were in the range of 1.92 p.u. up to 3.83 p.u. The results of testing the algorithm for identification of the electrical parameters of power transformers in this article are presented for one of the most typical cases below (Figures 4-6) the calculated and averaged values of the inductance of power transformer TDC-80 000/110. 


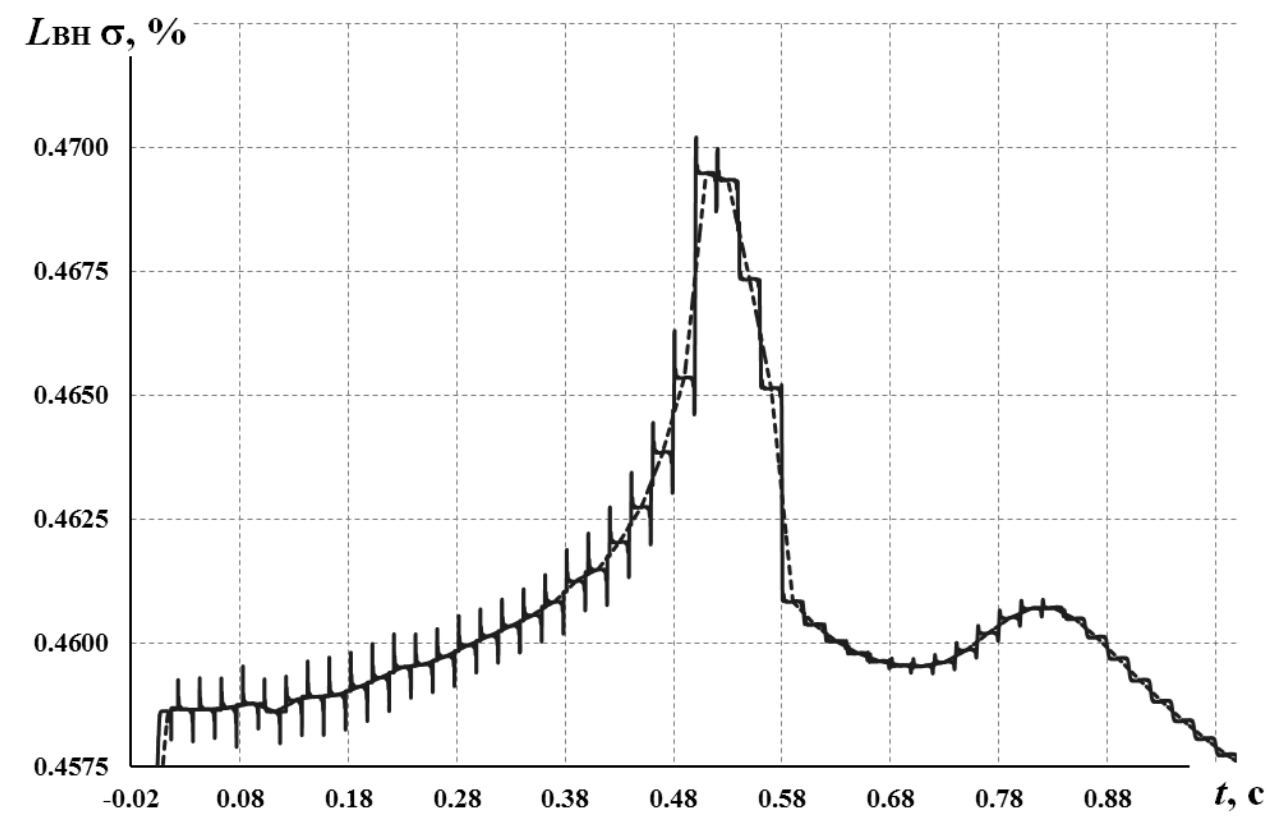

Fig. 4. Estimated (solid line) and averaged (dashed line) leakage inductance characteristics of the HV winding in identification of the electrical parameters of the TDC-80 000/110 transformer in idle mode

The analysis of the characteristics of the variation of the leakage inductances of the windings of the highest $\left(L_{1} \sigma\right)$ and lower $\left(L_{2} \sigma\right)$ voltages revealed the following features. The calculated and averaged characteristics of the leakage inductance of the winding of the highest voltage $\left(L_{1} \sigma\right)$ have a pronounced maximum at the time instant of about $0.5 \mathrm{~s}$ (Figure 4).

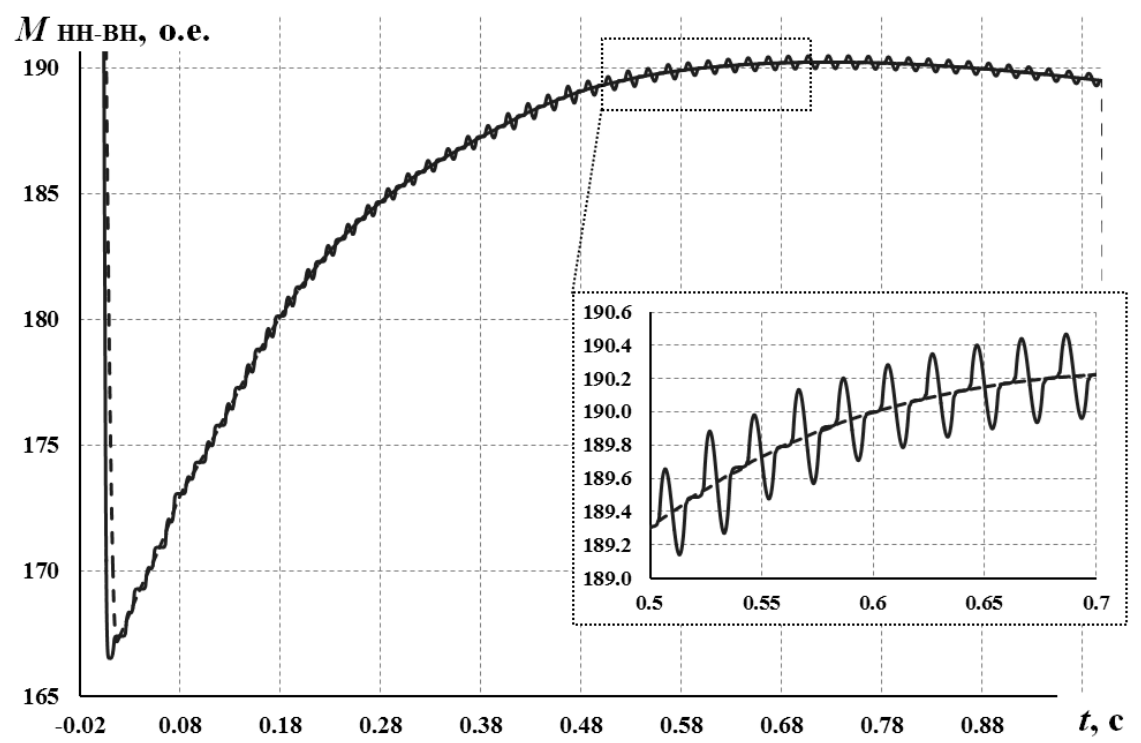

Fig. 5. Calculated (solid line) and averaged (dashed line) characteristics of the mutual inductance of 
the $\mathrm{HV}$ and LV windings in identification of the electrical parameters of the TDC-80 000/110 transformer in idle mode

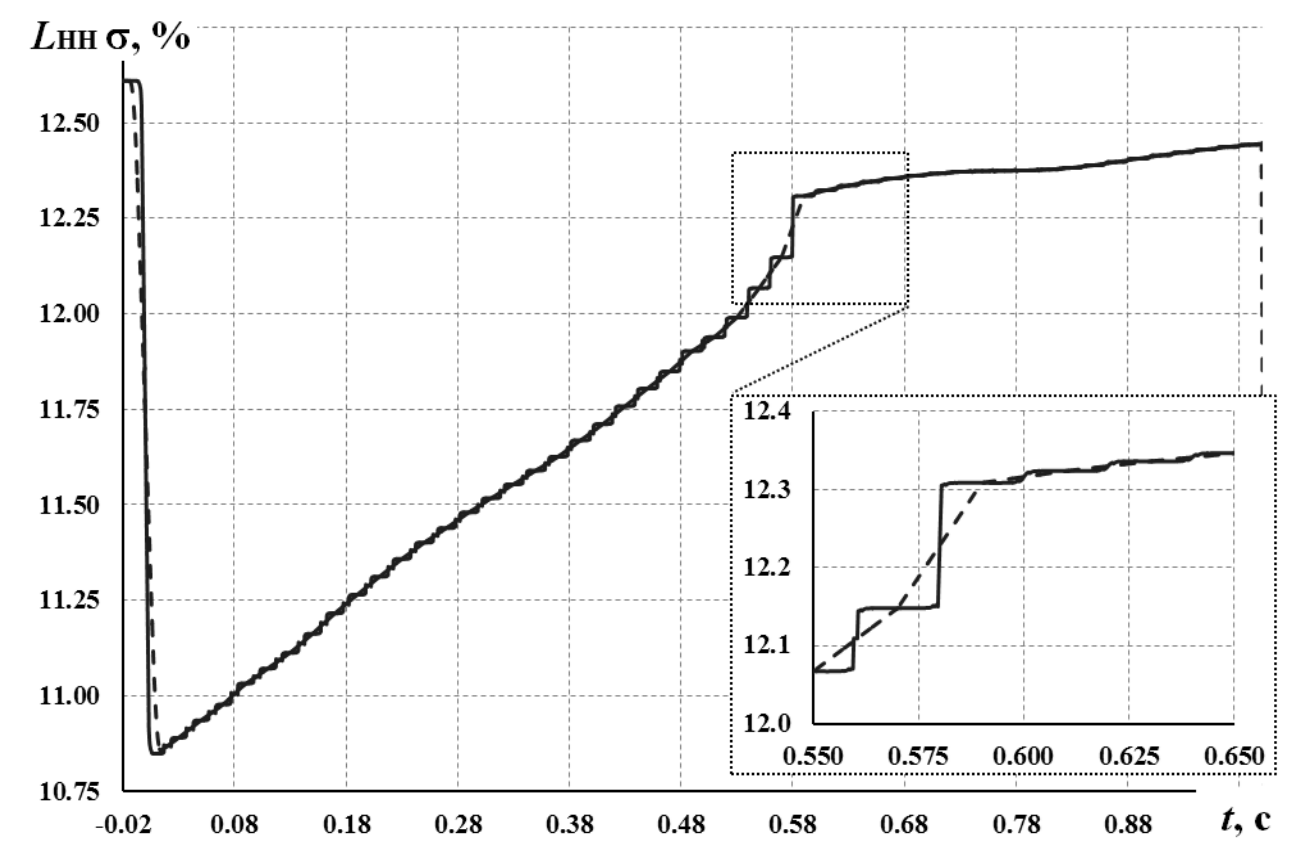

Fig. 6. Calculated (solid line) and averaged (dashed line) leakage inductance characteristics of the LV winding in identification of the electrical parameters of the TDC-80 000/110 transformer in idle mode

However, due to the fact that the change in the inductance of the winding of the highvoltage (HV) $L_{1} \sigma$ occurs in a small range, it can be considered almost constant and equal in size to $0.46 \%$ (Figure 4, Table 1, Line 6). The leakage inductance of the low-voltage (LV) winding $L_{2} \sigma$ (Figure 6) varies linearly over almost the entire interval $t \in[0 ; 0.58]$ of the non-stationary operation mode. Its minimum value $L_{2} \sigma=10.35 \%$ (Figure 6, Table 1, Line 6 ) is close in value to the short circuit voltage. After about $0.6 \mathrm{~s}$ after the switching on the transformer, the change in the leakage inductance of the LV winding occurs asymptotically, in value approximation of $12.3-12.4 \%$ (Figure 6, Table 1, Line 6).

Table 1. Averaged results of parametric identification of the power transformers in non-stationary modes of switching on idling

\begin{tabular}{|c|c|c|c|c|c|c|c|c|}
\hline \multirow{3}{*}{$\begin{array}{c}\text { Transformer } \\
\text { brand }\end{array}$} & \multicolumn{6}{|c|}{ Averaged identification parameters } & \multirow{2}{*}{\multicolumn{2}{|c|}{$\begin{array}{c}\text { Passport } \\
\text { parameters }\end{array}$}} \\
\hline & \multicolumn{2}{|c|}{$L_{1 \sigma}, \%$} & \multicolumn{2}{|c|}{$M_{21}$, o.e. } & \multicolumn{2}{|c|}{$L_{2} \sigma, \%$} & & \\
\hline & $\min$ & $\max$ & $\min$ & $\max$ & $\min$ & $\max$ & $I_{\mathrm{UL}}, \%$ & $U_{\mathrm{SC}}, \%$ \\
\hline Trihal $2500 / 10$ & 0.84 & 0.96 & 83.27 & 112.21 & 4.55 & 6.38 & - & 6.0 \\
\hline TD-40 000/110 & 0.62 & 0.81 & 138.11 & 184.45 & 9.28 & 11.56 & 0.65 & 11.0 \\
\hline TDC-80 000/110 & 0.457 & 0.47 & 166.25 & 190.20 & 10.35 & 12.38 & 0.55 & 11.0 \\
\hline $\begin{array}{l}\text { TDC- } \\
125000 / 110\end{array}$ & 0.54 & 0.58 & 173.15 & 194.17 & 10.15 & 11.77 & 0.60 & 11.0 \\
\hline $\begin{array}{l}\text { TC- } \\
630000 / 330\end{array}$ & 0.24 & 0.37 & 274.34 & 312.71 & 11.76 & 13.24 & 0.35 & 11.5 \\
\hline
\end{tabular}


The mutual induction of the LV and HV windings $\left(M_{21}\right)$, on the contrary, varies over a fairly wide range. Its minimum value in the initial stage of the transient is about 166 p.u. (Figure 5, table 1, line 6). In the final stage of the non-stationary mode, the mutual inductance of the transformer windings $M_{21}$ changes asymptotically and tends to values around 190 p.u. (table 1, line 6), which almost corresponds to the value of the steady-state current of idling $I_{\mathrm{XX}}=0.55 \%$ (table 1 , line 6 ).

Characteristics of changes of identifiable electrical parameters of power transformers Trihal $2500 / 10, \quad$ TD-40 000/110, TDC-125000/110 and TC-630 000/330 in non-stationary modes of their switching on idling are almost identical. However, it should be noted that in other cases, the asymptotic change in the calculated and averaged characteristics of the identification parameters change of power transformers is observed somewhat earlier - at a time of about $0.3 \mathrm{~s}$. This physical phenomenon is explained by a reduced (in comparison with the above-described results) level of switching currents and, as a result, a less saturated state of the transformer.

Based on the above, an important practical conclusion follows that for efficient and high-quality control of the magnetization current in the sub-transient (initial) stages of nonstationary idling modes, a refined mathematical description of power transformer equipment is required, since its calculated equivalent circuit with passport characteristics does not meet high accuracy requirements. Considering this, the results of the study of adaptive compensation of magnetization currents of power transformers and displacement currents, which are typical for the extended intersystem (interstate) connections, are presented in [4-10]. Unlike with the currently existing stationary compensation solutions in [4-17], the use of additional software algorithms for identification of power equipment parameters is proposed. The proposed method allows to reduce the minimum tripping current to the level of $0.1-0.15$ p.u. and to implement a dynamic correction of the tripping with a minimum level of brake signals ( $\mathrm{Kt}=0.1-0.15$ p.u. $)$.

The industrial introduction of such highly sensitive digital protection systems with a speed equal to the period of the industrial frequency will significantly improve the stability of the interconnected power systems and minimize the harm from damages of the protected equipment.

\section{Conclusion}

1. A methodology and software modules for identification of electromagnetic parameters of power transformers have been developed. As a result of the assessment of the sensitivity of identification equations of the power equipment parameters, it is shown that the surface of the objective function always has a global minimum, which identifies the existence and uniqueness of the solution of the system in the space of identifications parameters.

2. It is established that the developed software algorithm is especially sensitive to the assignment of zero initial values of parameters $R_{\mu}, L_{\mu}$ of the magnetization branch of power transformers. In the latter case, the root-mean-square error of the identification equations can reach critical (up to 250 p.u.) values in terms of speed and stable convergence of the numerical method. Positive non-zero initial conditions are characterized by monotonously decreasing vector function of the root-mean-square error $E(X)$.

3 . The weak sensitivity of the iterative process of identification the longitudinal parameters $R$ and $L$ to the assignment of them with zero initial values, at which the rootmean-square error is less than $3 \%$, is revealed. Similar indexes of the algorithm sensitivity were detected in assignment of zero initial values of the electrical parameters $R_{1}$ and $L_{1} \sigma$ of the primary winding of a two-winding transformer. Moreover, throughout the entire 
domain, the vector function $E(X)$ is positive and decreases to the convergence point of the system of equations. Near the extremum, the surface $E(X)$ is almost uniform and linear.

4. As a result of full-scale experiments, the approbation of the developed algorithm for identification of electrical parameters of three-phase power two-winding transformers with a rated voltage of $330 \mathrm{kV}, 110 \mathrm{kV}$ and $10 \mathrm{kV}$ in non-stationary operation modes was carried out. It is established that the calculated and averaged characteristics of the leakage inductances of the HV $\left(L_{1} \sigma\right)$ and $\operatorname{LV}\left(L_{2} \sigma\right)$ windings in the sub-transient (initial) stage of the non-stationary switching mode of power three-phase transformers are almost linear.

The mutual inductance of the LV and HV windings $\left(M_{21}\right)$ of three-phase power transformers varies asymptotically in a fairly wide range up to $\pm 15 \%$ regarding the steadystate values. In the final stage of the non-stationary mode, the mutual inductance of the transformer windings $M_{21}$ tends to values inversely proportional to the current value of steady-state idle mode.

5. The impossibility of applicability the linear calculated equivalent circuits of transformer equipment, obtained based on its passport characteristics, is substantiated. It is shown, that in the sub-transient (initial) stages of non-stationary modes, the reduced value of the error in calculating the currents of power transformers with the use of their passport characteristics, can reach $10-15 \%$. For effective and high-quality reproduction of magnetizing currents of power equipment, a refined mathematical description is required taking into account the nonlinearity of its characteristics.

\section{References}

1. I.G. Karapetyan, Handbook of electrical network design, ENAS, 392 (2009)

2. D.L. Faibisovich, Handbook of electrical network design - 3d edition, revision and addition, ENAS, 300 (2009)

3. A.M. Kontorovich, The method of calculating the modes and static stability of complex electrical systems with the frequency change: dissertation of the candidate of technical Sciences: 05.14.02, 216 (1979)

4. V.K. Vanin, News of higher educational institutions. Electromechanics 5, 55-62 (2011)

5. V.K. Vanin, M.G. Popov, S.O. Popov, Patent of invention №2538214 (19.06.2013). Published 18.11.2014, Report №10.

6. M.G. Popov, Power Technology and Engineering 50(1), 87-92 (2016)

7. M.G. Popov, News of higher educational institutions. Electromechanics 60(6), 91-96 (2017)

8. M.G. Popov, "Relay Protection and Automation" - scientific and practical publication 01(30), 39-45 (2018)

9. M.G. Popov, Scientific and technical statements SPbPU. Natural and engineering sciences 24(1), 17-26 (2018)

10. M.G. Popov, Modern devices of emergency control of interconnected power systems: autoabstract, dissertation of the candidate of technical Sciences: 05.14.02, 39-41 (2018)

11. A.V. Gudkov, D.N. Dadonov, E.A. Krotkov, V.E. Shchepinin, N.I. Didenko, V. Parkhomenko, 2017 6th International Conference on Reliability, Infocom Technologies and Optimization: Trends and Future Directions, ICRITO 2017 2018Janua, 182-187 (2018), doi:10.1109/ICRITO.2017.8342421 
12. V.M. Barinov, D.V. Kiesewetter, 10th International Conference - 2016 Electric Power Quality and Supply Reliability, PQ 2016, Proceedings 55-58 (2016), doi:10.1109/PQ.2016.7724089

13. A. Lyamov, M. Makarova, S. Smolovik, 2015 IEEE Eindhoven PowerTech, PowerTech 2015 (2015), doi:10.1109/PTC.2015.7232543

14. D.V. Kiesewetter, N.M. Zhuravleva, A.S. Reznik, A.V. Tukacheva, E.G. Smirnova, A.K. Khripunov, 10th International Conference - 2016 Electric Power Quality and Supply Reliability, PQ 2016, Proceedings 193-198 (2016), doi:10.1109/PQ.2016.7724112

15. A. Bolotin, V. Bakayev, S. Vazhenin, Journal of Physical Education and Sport 16, 102-108 (2016), doi:10.7752/jpes.2016.01017

16. N. Zhuravleva, A. Reznik, D. Kiesewetter, A. Tukacheva, E. Smirnova, 2016 57th International Scientific Conference on Power and Electrical Engineering of Riga Technical University, RTUCON 2016 (2016), doi:10.1109/RTUCON.2016.7763089

17. N. Zhuravleva, A. Reznik, D. Kiesewetter, D. Tashlanov, Proceedings of the 2017 IEEE Russia Section Young Researchers in Electrical and Electronic Engineering Conference, ElConRus 2017, 1220-1223 (2017), doi:10.1109/EIConRus.2017.7910781

18. N. Zhuravleva, A. Reznik, A. Tukacheva, D. Kiesewetter, E. Smirnova, Proceedings of the 2016 IEEE North West Russia Section Young Researchers in Electrical and Electronic Engineering Conference, EIConRusNW 2016, 747-751 (2016), doi:10.1109/EIConRusNW.2016.7448288 